\title{
An efficient approach for solution of fractional-order Helmholtz equations
}

\author{
Nehad Ali Shah ${ }^{1,2^{*}}$, Essam R. El-Zahar ${ }^{3,4}$, Mona D. Aljoufi ${ }^{5}$ and Jae Dong Chung ${ }^{6}$
}

"Correspondence:

nehad.ali.shah@tdtu.edu.vn

'Informetrics Research Group, Ton

Duc Thang University, Ho Chi Minh

City, Vietnam

${ }^{2}$ Faculty of Mathematics \& Statistics,

Ton Duc Thang University, Ho Chi

Minh City, Vietnam

Full list of author information is

available at the end of the article

\section{Springer}

\begin{abstract}
In this article, a hybrid technique called the homotopy perturbation Elzaki transform method has been implemented to solve fractional-order Helmholtz equations. In the hybrid technique, the Elzaki transform method and the homotopy perturbation method are amalgamated. Three problems are solved to validate and demonstrate the efficacy of the present technique. It is also demonstrated that the results obtained from the suggested technique are in excellent agreement with the results by other techniques. It is shown that the proposed method is efficient, reliable and easy to implement for various related problems of science and engineering.
\end{abstract}

Keywords: Elzaki transform; Homotopy perturbation method; Helmholtz equations; Mittag-Leffler function

\section{Introduction}

In this century, the study of fractional calculus and its various applications in mathematics, physics and engineering have received considerable attention. Fractional calculus applications are found in many areas, such as electrical networks, dynamic device control theory, probability and statistics, corrosion electrochemistry, chemical mechanics, and optics and signal processing. Linear or nonlinear fractional-order differential equations may be successfully modeled. A fractional differential equation is obtained from the classical diffusion equation of mathematical physics by replacing the $n$th order time derivative with a fractional-order derivative $\gamma(n-1<\gamma \leq n)$, which is now the area of increasing interest apparent in the literature study. A significant phenomenon of these evolution equations is that it produces the Brownian fractional movement, a Brownian motion generalization. In several articles and books, different definitions of fractional calculus are available [1-10].

The Helmholtz equation or the reduced wave equation is an elliptic partial differential equation (PDE) derived directly from the wave model. A Helmholtz equation is a PDE that represents a time-independent mechanical development in space. The Helmholtz equation is one of the most significant in physics and applied mathematical models [10]. The Helmholtz equation's solutions, which are generally generated from the separation of variables, address important science phenomena. The equations occur in some phenomena, such as electromagnetic waves in fluids, vibrating lines, plates, walls, acoustics, magnetic fields, nuclear plants and the Lamb equation in geoscience. Consider a two-dimensional

(c) The Author(s) 2021. This article is licensed under a Creative Commons Attribution 4.0 International License, which permits use sharing, adaptation, distribution and reproduction in any medium or format, as long as you give appropriate credit to the original author(s) and the source, provide a link to the Creative Commons licence, and indicate if changes were made. The images or other third party material in this article are included in the article's Creative Commons licence, unless indicated otherwise in a credit line to the material. If material is not included in the article's Creative Commons licence and your intended use is not permitted by statutory regulation or exceeds the permitted use, you will need to obtain permission directly from the copyright holder. To view a copy of this licence, visit http://creativecommons.org/licenses/by/4.0/. 
non-homogeneous isotropic medium whose velocity is $c$ in euclidean space $[11,12]$. The wave result is $\mu(x, y)$ corresponding to the harmonic origin $\phi(x, y)$ vibrating at the specified fixed frequency $\omega>0$ satisfies the Helmholtz equation for the defined area $R$ :

$$
\frac{\partial^{2} \mu(x, y)}{\partial x^{2}}+\frac{\partial^{2} \mu(x, y)}{\partial y^{2}}+\lambda \mu(x, y)=-\phi(x, y)
$$

where $\mu(x, y)$ is an appropriately differentiable function at the boundary of $R$, and $\phi(x, y)$ is a specified function, $\lambda>0$ is a constant value, and $\sqrt{\lambda}=\frac{\omega}{c}$ is a wave number with a wavelength of $\frac{2 \pi}{\sqrt{\lambda}}$. If $\phi(x, y)=0$ is necessary, then Eq. (1), the Helmholtz equation, is homogeneous. Most equations connected to steady-state oscillations (electromagnetic, acoustic, hydraulic, thermoelectric) lead to a two-dimensional Helmholtz equation. If the positive sign (in front of the $\lambda$ term) is modified to a negative sign, this problem defines mass transport procedures with first-order volume chemical compounds. For instance, in linear acoustics, $\phi(x, y)$ can reflect a disturbance of the reference state pressure (Thompson and Pinsky, 1995 [12]). Conservation equations, which are also transformed into Helmholtz equations, often have various implementations in several physical problems, such as shear viscosity streams or fluids constrained inside thermophysical walls [11-14]. In recent decades, many of the numerical and analytical techniques have been implemented to solve fractional-order Helmholtz equations, such as He's homotopy perturbation technique [10], Laplace variational iteration technique [15], the reduced differential transform technique [16], the q-homotopy analysis transform technique [17], the spectral technique [17] and He's variational iteration technique [18].

The homotopy perturbation method (HPM), first suggested by the Chinese scientist $\mathrm{He}$ has played a crucial role since 1998 [19]. This is so because of the idea that it approaches the scheme, not requiring any discrimination or linearization. This technique is fair, efficient and effective, and eliminates an unconditioned matrix, infinite series and complicated integrals. This algorithm does not need a specific parameter of the problem. In this technique, according to the homotopy method, a homotopy with an embedding parameter $p \in[0,11]$ is constructed, and the embedding parameter is considered as a "small parameter," which can take full advantage of the traditional perturbation techniques and homotopy methods [20-23]. The Ezaki transformation (E.T.) is a recent integral transform implemented in 2010 by Elzaki. E.T. is a modified transformation of the Laplace and Sumudu transformations. It is worth noting that absolute differential equations with variable coefficients cannot be achieved by Laplace and Sumudu transformations but can be easily handled with the use of E.T. [24-26]. The homotopy perturbation Elzaki transformation method (HPETM) combines the Elzaki transformation and the homotopy perturbation method. Many researchers have solved different equations with the help of HPETM, such as the Navier-Stokes equations [27], heat-like equations [28], a gas dynamic equation [29], a hyperbolic equation and Fisher's equation [30].

As the main aim of this work the HPETM is implemented to solve fractional-order twodimensional Helmholtz equations. The HPETM solutions are determined for three particular examples of fractional-order two-dimensional Helmholtz equations. The higher efficiency and accuracy of HPETM is observed, using graphs comparing to exact solutions. The series form solutions of HPETM for fractional-order Helmholtz equations have shown the desired rate of convergence. Thus, the present technique is proposed to solve 
other fractional partial differential equations. The article is organized as follows: after presenting basic definitions and fractional calculus properties in Sect. 2, we introduce the proposed method in Sect. 3. Section 4 presents the exact and approximate solutions of three examples of fractional Helmholtz equation. Section 5 concludes the study.

\section{Preliminaries concepts}

Definition 2.1 ([24-26]) Let us write the definition of the gamma function:

$$
\Gamma(\gamma)=\int_{0}^{\infty} e^{-\eta} \eta^{\gamma-1} \partial \eta, \quad \gamma>0 .
$$

Definition 2.2 A real function $g(x), x>0$, is said to be in the space $C_{\gamma}, \gamma \in R$ if there exists a real number $p>\gamma$, such that $g\left(x=x^{p} g(x)\right)$, where $g(x) \in[0, \infty]$ and it is said to be in space $C_{\gamma}^{\kappa}$ if $g^{(\kappa)} \in C_{\gamma}, \kappa \in N[24-26]$.

Definition 2.3 (The partial fractional-order derivatives [24-26]) Now consider $g(x)$ to be a function of $n$ variables $x_{i}, i=1, \ldots, \kappa$, also of group $C$ on $D \in R_{\kappa}$. As an implement of definition 2 we define the fractional order $\gamma$ for $g(x)$ with respect to $x_{i}$ by

$$
\partial_{x}^{\gamma} g=\left.\frac{1}{\Gamma(\kappa-a)} \int_{0}^{x_{i}}\left(x_{i}-1\right)^{\kappa-\gamma-1} \partial_{x_{i}}^{\gamma} g\left(x_{j}\right)\right|_{x_{j}=\eta} d \eta \text {. }
$$

Definition 2.4 The Riemann-Liouville fractional-order equation, $\gamma>0$, of a function $f \in$ $C_{\kappa}, \gamma \geq-1$, is defined as $[1,2,24-26]$

$$
\begin{aligned}
& J^{\gamma} g(x)=\frac{1}{\Gamma(\gamma)} \int_{0}^{x}(x-1)^{\gamma-1} g(\eta) \partial \eta, \gamma, \quad x>0, \\
& J^{\gamma} g(x)=g(x) .
\end{aligned}
$$

Some properties of the operator are:

$$
\begin{aligned}
& \text { For } g \in C_{\kappa}, \gamma \geq-1, \gamma, \delta \geq 0 \text { and } \alpha>-1 \\
& \begin{array}{l}
J^{\gamma} J^{\delta} g(x)=J^{\gamma+\delta} g(x), \\
J^{\gamma} J^{\delta} g(x)=J^{\delta} J^{\gamma} g(x), \\
J^{\gamma} x^{\delta}=\frac{\Gamma(\delta+1)}{(\gamma+\delta+1)} x^{\gamma+\delta} .
\end{array}
\end{aligned}
$$

Lemma 2.5 If $\kappa-1<\gamma \leq \kappa, \kappa \in N$ and $g \in C_{\kappa}, \gamma \geq-1$, then $D^{\gamma} J^{\gamma} g(x)=g(x)$ and

$$
D^{\gamma} J^{\gamma} g(x)=g(x)-\sum_{k=0}^{m-1} g^{(k)}(0) \frac{x^{k}}{k !}, \quad x>0 .
$$

\section{Fundamental concept of the Elzaki transformation}

A new transformation called the Elzaki transformation is described for the exponential order function that we find in the $A$ set, represented by [24-26]

$$
A=g(\eta): \ni\left|M, k_{1}, k_{2}>0,\right| g(\eta) \mid<M e^{\frac{|\eta|}{k_{j}}}, \quad \text { if }(\eta) \in(-1)^{j} \times[0, \infty) .
$$


The constant $M$ must be a finite number, $k 1$ and $k 2$ are finite or infinite for a given function in the set. The Elzaki transformation is described through the integral equation

$$
E[g(\eta)]=T(s)=s \int_{0}^{\infty} g(\eta) e^{\frac{-\eta}{s}} d \eta, \quad \eta \geq 0, k_{1} \leq s \leq k_{2} .
$$

We obtain the following result from the description and the fundamental analyses:

$$
\begin{aligned}
& E\left[\eta^{n}\right]=n ! s^{n+2}, \\
& E\left[g^{\prime}(\eta)\right]=\frac{T(s)}{s}-s g(0), \\
& E\left[g^{\prime \prime}(\eta)\right]=\frac{T(s)}{s^{2}}-g(0)-s g^{\prime}(0), \\
& E\left[g^{(n)}(\eta)\right]=\frac{T(s)}{s^{n}}-\sum_{k=0}^{n-1} s^{2-n+k} g^{(k)}(0) .
\end{aligned}
$$

Theorem 2.6 If $T(s)$ is the Elzaki transformation of $(\eta)$, the Elzaki transformation of the Riemann-Liouville derivative can be taken into consideration as follows [24-26]:

$$
E\left[D^{\gamma} g(\eta)\right]=s^{-\gamma}\left[T(s)-\sum_{k=1}^{n}\left\{D^{\gamma-k} g(0)\right\} ; \quad-1<n-1 \leq \gamma<n .\right.
$$

Proof Let us take the Laplace transform of

$$
\begin{aligned}
& g^{\prime}(\eta)=\frac{d}{d \eta} g(\eta), \\
& L\left[D^{\gamma} g(\eta)\right]=S^{\gamma} T(s)-\sum_{k=0}^{n-1} s^{k}\left[D^{\gamma-k-1} g(0)\right] \\
& =s^{\gamma} T(s)-\sum_{k=0}^{n-1} s^{k-1}\left[D^{\gamma-k} g(0)\right]=s^{\gamma} T(s)-\sum_{k=0}^{n-1} s^{k-2}\left[D^{\gamma-k} g(0)\right] \\
& =s^{\gamma} T(s)-\sum_{k=0}^{n-1} \frac{1}{s^{-k+2}}\left[D^{\gamma-k} g(0)\right]=s^{\gamma} T(s)-\sum_{k=0}^{n-1} \frac{1}{s^{\gamma-k+2-\gamma}}\left[D^{\gamma-k} g(0)\right] \\
& =s^{\gamma} T(s)-\sum_{k=0}^{n-1} s^{\gamma} \frac{1}{s^{\gamma-k+2}}\left[D^{\gamma-k} g(0)\right], \\
& L\left[D^{\gamma} g(\eta)\right]=s^{\gamma}\left[T(s)-\sum_{k=0}^{n-1}\left(\frac{1}{s}\right)^{\gamma-k+2}\left[D^{\gamma-k} g(0)\right]\right] .
\end{aligned}
$$

Therefore, when we put $\frac{1}{s}$ for $s$, the Elzaki transform of fractional order of $g(\eta)$ becomes

$$
E\left[D^{\gamma} g(\eta)\right]=s^{-\gamma}\left[T(s)-\sum_{k=0}^{n}(s)^{\gamma-k+2}\left[D^{\gamma-k} g(0)\right]\right] .
$$


Definition 2.7 The Caputo fractional-order Elzaki transformation by using Theorem 2.6 is given as [1-3]

$$
E\left[D_{\eta}^{\gamma} g(\eta)\right]=s^{-\gamma} E[g(\eta)]-\sum_{k=0}^{\kappa-1} s^{2-\gamma+k} g^{(k)}(0), \quad \text { where } \kappa-1<\gamma<\kappa .
$$

\section{Homotopy perturbation Elzaki transform method}

Being the basic concept of the given technique, we consider a general form of PDE:

$$
\begin{aligned}
& D_{y}^{\gamma} \mu(x, y)+M \mu(x, y)+N \mu(x, y)=h(x, y), \quad y>0,0<\gamma \leq 1, \\
& \mu(x, 0)=g(x), \quad v \in \Re .
\end{aligned}
$$

Applying the Elzaki transformation of Eq. (3)

$$
\begin{aligned}
& E\left[D_{y}^{\gamma} \mu(x, y)+M \mu(x, y)+N \mu(x, y)\right]=E[h(x, y)], \quad y>0,0<\gamma \leq 1, \\
& \mu(x, y)=s^{2} g(x)+s^{\gamma} E[h(x, y)]-s^{\gamma} E[M \mu(x, y)+N \mu(x, y)] .
\end{aligned}
$$

Now, by taking the inverse Elzaki transformation, we have

$$
\mu(x, y)=F(x, y)-E^{-1}\left[s^{\gamma} E\{M \mu(x, y)+N \mu(x, y)\}\right],
$$

where

$$
F(x, y)=E^{-1}\left[s^{2} g(x)+s^{\gamma} E[h(x, y)]\right]=g(v)+E^{-1}\left[s^{\gamma} E[h(x, y)]\right] .
$$

Now, the perturbation procedure in terms of a power series with parameter $p$ is presented as

$$
\mu(x, y)=\sum_{k=0}^{\infty} p^{k} \mu_{k}(x, y)
$$

where $p$ is the perturbation parameter and $p \in[0,1]$.

The non-linear term can be defined as

$$
N \mu(x, y)=\sum_{k=0}^{\infty} p^{k} H_{k}\left(\mu_{k}\right)
$$

where $H_{n}$ are He's polynomials in terms of $\mu_{0}, \mu_{1}, \mu_{2}, \ldots, \mu_{n}$, and can be determined as

$$
H_{n}\left(\mu_{0}, \mu_{1}, \ldots, \mu_{n}\right)=\frac{1}{\gamma(n+1)} D_{p}^{k}\left[N\left(\sum_{k=0}^{\infty} p^{k} \mu_{k}\right)\right]_{p=0},
$$

where $D_{p}^{k}=\frac{\partial^{k}}{\partial p^{k}}$.

Substituting Eqs. (8) and (9) in Eq. (5), we get

$$
\sum_{k=0}^{\infty} p^{k} \mu_{k}(x, y)=F(x, y)-p \times\left[E^{-1}\left\{s^{\gamma} E\left\{M \sum_{k=0}^{\infty} p^{k} \mu_{k}(x, y)+\sum_{k=0}^{\infty} p^{k} H_{k}\left(\mu_{k}\right)\right\}\right\}\right] .
$$


On both sides, by comparison of the coefficients $p$, we have

$$
\begin{aligned}
& p^{0}: \mu_{0}(x, y)=F(x, y), \\
& p^{1}: \mu_{1}(x, y)=E^{-1}\left[s^{\gamma} E\left(M \mu_{0}(x, y)+H_{0}(\mu)\right)\right], \\
& p^{2}: \mu_{2}(x, y)=E^{-1}\left[s^{\gamma} E\left(M \mu_{1}(x, y)+H_{1}(\mu)\right)\right], \\
& \vdots \\
& p^{k}: \mu_{k}(x, y)=E^{-1}\left[s^{\gamma} E\left(M \mu_{k-1}(x, y)+H_{k-1}(\mu)\right)\right], \quad k>0, k \in N .
\end{aligned}
$$

The $\mu_{k}(x, y)$ component can be determined easily which quickly leads us to the convergent series. We can get, as $p \rightarrow 1$,

$$
\mu(x, y)=\lim _{M \rightarrow \infty} \sum_{k=1}^{M} \mu_{k}(x, y)
$$

\section{Applications}

In this section of the article, the method HPETM will be applied to some examples to understand the proposed method's procedure. Some numerical simulations are carried out to visualize the family of Helmholtz equations through HPETM.

Example 1 Consider the fractional-order Helmholtz equations

$$
\frac{\partial^{\gamma} \mu(x, y)}{\partial x^{\gamma}}+\frac{\partial^{2} \mu(x, y)}{\partial y^{2}}-\mu(x, y)=0, \quad 1<\gamma \leq 2
$$

with initial condition

$$
\mu(0, y)=y \quad \text { and } \quad \mu_{x}(0, y)=0 .
$$

Applying the Elzaki Transformation of Eq. (13), we get

$$
\begin{aligned}
& \frac{1}{s^{\gamma}} E[\mu(x, y)]=\mu(0, y) s^{2-\gamma}-E\left\{\frac{\partial^{2} \mu(x, y)}{\partial y^{2}}-\mu(x, y)\right\}, \\
& E[\mu(x, y)]=s^{2} \mu(0, y)-s^{\gamma} E\left\{\frac{\partial^{2} \mu(x, y)}{\partial y^{2}}-\mu(x, y)\right\} .
\end{aligned}
$$

Taking the inverse Elzaki transformation, we have

$$
E[\mu(x, y)]=y-E^{-1}\left[s^{\gamma} E\left\{\frac{\partial^{2} \mu(x, y)}{\partial y^{2}}-\mu(x, y)\right\}\right] .
$$

Implementing HPM in Eq. (17), we obtain

$$
\sum_{\kappa=0}^{\infty} p^{\kappa} \mu_{\kappa}(x, y)=y-p\left[E^{-1}\left\{s^{\gamma} E\left\{\left(\sum_{\kappa=0}^{\infty} p^{\kappa} \mu_{\kappa}(x, y)\right)_{y y}-\sum_{\kappa=0}^{\infty} p^{\kappa} \mu_{\kappa}(x, y)\right\}\right\}\right] .
$$


On comparing the coefficients $p$ on both sides, we obtain

$$
\begin{aligned}
& p^{0}: \mu_{0}(x, y)=y, \\
& p^{1}: \mu_{1}(x, y)=-E^{-1}\left[s^{\gamma} E\left\{\frac{\partial^{2} \mu_{0}(x, y)}{\partial y^{2}}-\mu_{0}(x, y)\right\}\right]=\frac{x^{\gamma}}{\Gamma(\gamma+1)} y, \\
& p^{2}: \mu_{2}(x, y)=-E^{-1}\left[s^{\gamma} E\left\{\frac{\partial^{2} \mu_{1}(x, y)}{\partial y^{2}}-\mu_{1}(x, y)\right\}\right]=\frac{x^{2 \gamma}}{\Gamma(2 \gamma+1)} y, \\
& p^{3}: \mu_{3}(x, y)=-E^{-1}\left[s^{\gamma} E\left\{\frac{\partial^{2} \mu_{2}(x, y)}{\partial y^{2}}-\mu_{2}(x, y)\right\}\right]=\frac{x^{3 \gamma}}{\Gamma(3 \gamma+1)} y, \\
& p^{4}: \mu_{4}(x, y)=-E^{-1}\left[s^{\gamma} E\left\{\frac{\partial^{2} \mu_{3}(x, y)}{\partial y^{2}}-\mu_{3}(x, y)\right\}\right]=\frac{x^{4 \gamma}}{\Gamma(4 \gamma+1)} y,
\end{aligned}
$$

The series form a solution of the given example, and we have

$$
\begin{aligned}
& \mu(x, y)=\mu_{0}(x, y)+\mu_{1}(x, y)+\mu_{2}(x, y)+\mu_{3}(x, y)+\mu_{4}(x, y)+\cdots \\
& \mu(x, y)=y\left[1+\frac{x^{\gamma}}{\Gamma(\gamma+1)}+\frac{x^{2 \gamma}}{\Gamma(2 \gamma+1)}+\frac{x^{3 \gamma}}{\Gamma(3 \gamma+1)}+\frac{x^{4 \gamma}}{\Gamma(4 \gamma+1)}+\cdots\right] .
\end{aligned}
$$

The exact result of Example 1 when $\gamma=2$ is

$$
\mu(x, y)=y \cosh x
$$

In the same way the solution of $y$-space can be determined through HPETM as

$$
\frac{\partial^{\gamma} \mu(x, y)}{\partial y^{\gamma}}+\frac{\partial^{2} \mu(x, y)}{\partial x^{2}}-\mu(x, y)=0
$$

with proper initial value

$$
\mu(x, 0)=x
$$

Thus the result of Eq. (21) is obtained,

$$
\mu(x, y)=x\left(1+\frac{y^{\gamma}}{\Gamma(\gamma+1)}+\frac{y^{2 \gamma}}{\Gamma(2 \gamma+1)}+\frac{y^{3 \gamma}}{\Gamma(3 \gamma+1)}+\frac{y^{4 \gamma}}{\Gamma(4 \gamma+1)}+\cdots\right)
$$

in the case when $\gamma=2$, then the solution through HPETM is

$$
\mu(x, y)=x \cosh y .
$$

Figure 1 depicts solutions in a two-dimensional plot of exact and HPETM solutions in Figs. (a) and (b) for different values of $\gamma=2,1.9,1.8,1.7,1.6,1.5$ for $x \in[0 ; 1]$ and $y=1$. In Fig. 2, the three-dimensional plots of exact and HPETM solutions in Figs. (a) and (b), respectively, at $\gamma=2$ and the closed contact of the exact and HPETM solutions are analyzed. In Fig. 3, the graphs (c) and (d) represent the HPETM solutions at $\gamma=1.8$ and 


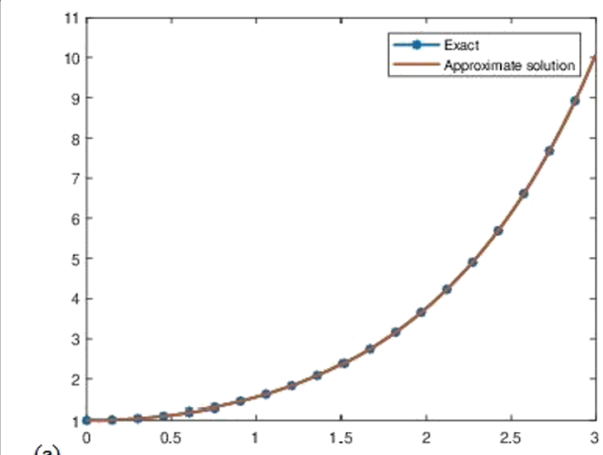

(a)

Figure 1 (a) Graphs of exact and approximate solution and (b) different fractional-order results of $\gamma$ of Example 1

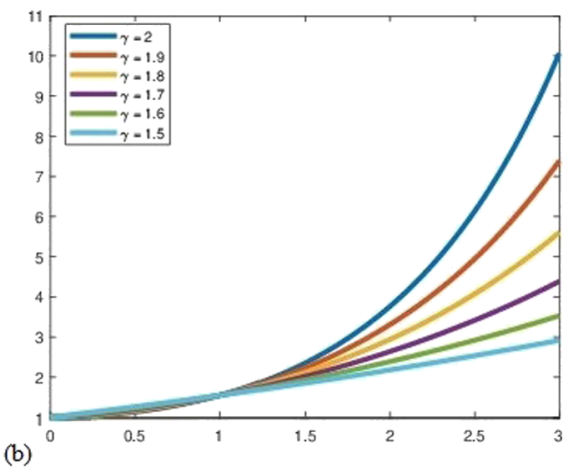

(b)

of

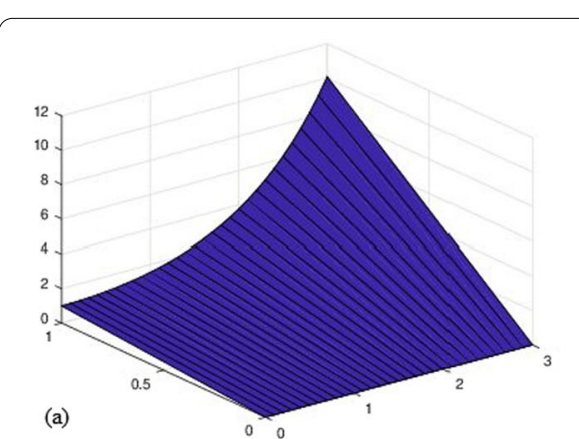

Figure 2 (a) Graphs of exact and (b) approximate solution of $\gamma=1$ of Example 1

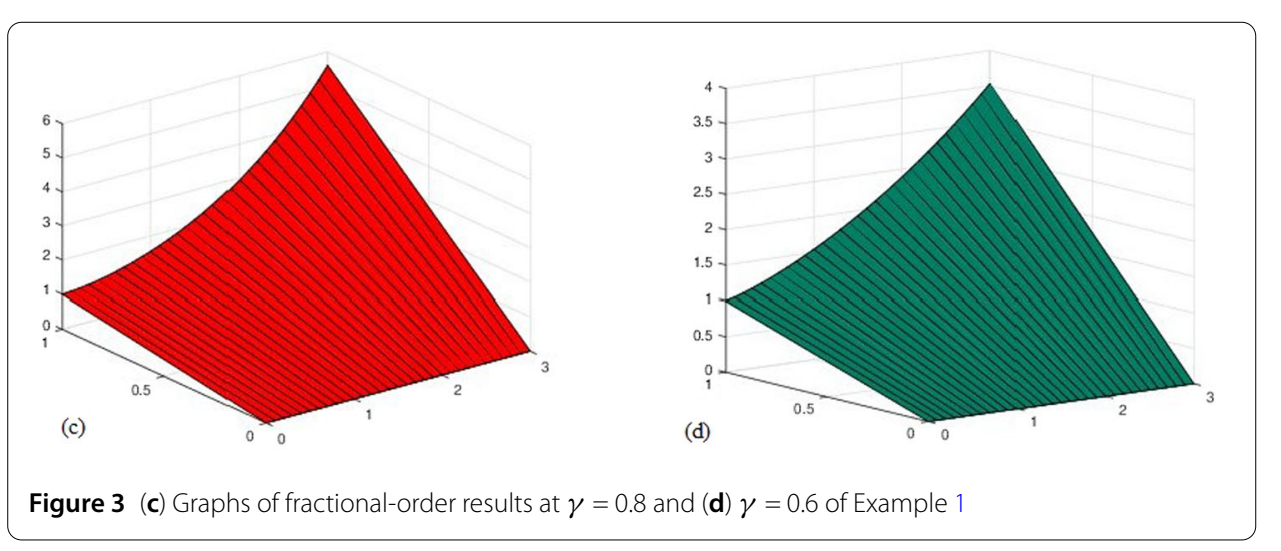

1.6, respectively, of Example 1. The fractional results are investigated to be convergent an integer-order result of each problem. In the same way, we can draw the figures for the $y$-space fractional derivative.

Example 2 Consider the fractional-order Helmholtz equation

$$
\frac{\partial^{\gamma} \mu(x, y)}{\partial x^{\gamma}}+\frac{\partial^{2} \mu(x, y)}{\partial y^{2}}+5 \mu(x, y)=0, \quad 1<\gamma \leq 2,
$$


Table 1 Comparison of HPETM and RDTM solutions of different fractional-order results at $\gamma$ of Example 1

\begin{tabular}{|c|c|c|c|c|c|}
\hline \multirow[t]{2}{*}{$\mu(x, y)$} & HPETM & HPETM & HPETM & RDTM & RDTM \\
\hline & $\gamma=1.5$ & $\gamma=1.7$ & $\gamma=2$ & $\gamma=1.7$ & $\gamma=2$ \\
\hline$(0.1,0.1)$ & $9.8994158 \times 10^{-4}$ & $1.8951426 \times 10^{-6}$ & $4.4682560 \times 10^{-8}$ & $1.8951426 \times 10^{-6}$ & $4.4682560 \times 10^{-8}$ \\
\hline$(0.2,0.2)$ & $9.5789042 \times 10^{-3}$ & $9.7686054 \times 10^{-6}$ & $9.8994158 \times 10^{-8}$ & $9.4137771 \times 10^{-6}$ & $9.7686054 \times 10^{-8}$ \\
\hline$(0.3,0.3)$ & $1.6770296 \times 10^{-3}$ & $1.9457270 \times 10^{-5}$ & $9.8994158 \times 10^{-7}$ & $1.8868189 \times 10^{-5}$ & $1.9457270 \times 10^{-7}$ \\
\hline$(0.4,0.4)$ & $1.9696739 \times 10^{-2}$ & $2.8187283 \times 10^{-5}$ & $2.9025947 \times 10^{-6}$ & $2.8187283 \times 10^{-5}$ & $2.9025947 \times 10^{-6}$ \\
\hline$(0.5,0.5)$ & $2.9389443 \times 10^{-2}$ & $3.7373595 \times 10^{-4}$ & $3.8474635 \times 10^{-6}$ & $3.7373595 \times 10^{-4}$ & $3.8474635 \times 10^{-6}$ \\
\hline$(0.6,0.6)$ & $3.8973948 \times 10^{-2}$ & $4.6424252 \times 10^{-4}$ & $4.7800451 \times 10^{-5}$ & $4.6424252 \times 10^{-4}$ & $4.7800451 \times 10^{-5}$ \\
\hline$(0.7,0.7)$ & $4.8445597 \times 10^{-2}$ & $5.5332903 \times 10^{-4}$ & $5.6998309 \times 10^{-5}$ & $5.5332903 \times 10^{-4}$ & $5.6998309 \times 10^{-5}$ \\
\hline$(0.8,0.8)$ & $5.7798601 \times 10^{-2}$ & $6.4090547 \times 10^{-4}$ & $6.6061216 \times 10^{-5}$ & $6.4090547 \times 10^{-4}$ & $6.6061216 \times 10^{-5}$ \\
\hline$(0.9,0.9)$ & $6.7025983 \times 10^{-2}$ & $7.2685898 \times 10^{-4}$ & $7.4980409 \times 10^{-5}$ & $7.2685898 \times 10^{-4}$ & $7.4980409 \times 10^{-5}$ \\
\hline$(1.0,1.0)$ & $7.6119511 \times 10^{-2}$ & $8.1105544 \times 10^{-4}$ & $8.3745391 \times 10^{-5}$ & $8.1105544 \times 10^{-4}$ & $8.3745391 \times 10^{-5}$ \\
\hline
\end{tabular}

with initial condition

$$
\mu(0, y)=y \quad \text { and } \quad \mu_{x}(0, y)=0 .
$$

Applying the Elzaki Transformation of Eq. (24), we get

$$
\begin{aligned}
& \frac{1}{s^{\gamma}} E[\mu(x, y)]=\mu(0, y) s^{2-\gamma}-E\left\{\frac{\partial^{2} \mu(x, y)}{\partial y^{2}}+5 \mu(x, y)\right\}, \\
& E[\mu(x, y)]=s^{2} \mu(0, y)-s^{\gamma} E\left\{\frac{\partial^{2} \mu(x, y)}{\partial y^{2}}+5 \mu(x, y)\right\} .
\end{aligned}
$$

Taking the inverse Elzaki transformation, we have

$$
E[\mu(x, y)]=y-E^{-1}\left[s^{\gamma} E\left\{\frac{\partial^{2} \mu(x, y)}{\partial y^{2}}+5 \mu(x, y)\right\}\right]
$$

By implementing the HPM in Eq. (28), we obtain

$$
\sum_{k=0}^{\infty} p^{k} \mu_{k}(x, y)=y-p\left[E^{-1}\left\{s^{\gamma} E\left\{\left(\sum_{k=0}^{\infty} p^{k} \mu_{k}(x, y)\right)_{y y}+5 \sum_{k=0}^{\infty} p^{k} \mu_{k}(x, y)\right\}\right\} .\right.
$$

On comparing the coefficients $p$ on both sides, we obtain

$$
\begin{aligned}
& p^{0}: \mu_{0}(x, y)=y, \\
& p^{1}: \mu_{1}(x, y)=-E^{-1}\left[s^{\gamma} E\left\{\frac{\partial^{2} \mu_{0}(x, y)}{\partial y^{2}}+5 \mu_{0}(x, y)\right\}\right]=-5 y \frac{x^{\gamma}}{\Gamma(\gamma+1)}, \\
& p^{2}: \mu_{2}(x, y)=-E^{-1}\left[s^{\gamma} E\left\{\frac{\partial^{2} \mu_{1}(x, y)}{\partial y^{2}}+5 \mu_{1}(x, y)\right\}\right]=25 y \frac{x^{2 \gamma}}{\Gamma(2 \gamma+1)}, \\
& p^{3}: \mu_{3}(x, y)=-E^{-1}\left[s^{\gamma} E\left\{\frac{\partial^{2} \mu_{2}(x, y)}{\partial y^{2}}+5 \mu_{2}(x, y)\right\}\right]=-125 \frac{x^{3 \gamma}}{\Gamma(3 \gamma+1)}, \\
& p^{4}: \mu_{4}(x, y)=-E^{-1}\left[s^{\gamma} E\left\{\frac{\partial^{2} \mu_{3}(x, y)}{\partial y^{2}}+5 \mu_{3}(x, y)\right\}\right]=625 y \frac{x^{4 \gamma}}{\Gamma(4 \gamma+1)},
\end{aligned}
$$


The series form a solution of the given example as

$$
\begin{aligned}
& \mu(x, y)=\mu_{0}(x, y)+\mu_{1}(x, y)+\mu_{2}(x, y)+\mu_{3}(x, y)+\mu_{4}(x, y)+\cdots \\
& \mu(x, y)=y\left[1-\frac{5 x^{\gamma}}{\Gamma(\gamma+1)}+\frac{25 x^{2 \gamma}}{\Gamma(2 \gamma+1)}-\frac{125 x^{3 \gamma}}{\Gamma(3 \gamma+1)}+\frac{625 x^{4 \gamma}}{\Gamma(4 \gamma+1)}+\cdots\right] .
\end{aligned}
$$

The exact result of Example 2, when $\gamma=2$, is

$$
\mu(x, y)=y \cos \sqrt{5} x
$$

In the same way, the solution of $y$-space can be determined through HPETM as

$$
\frac{\partial^{\gamma} \mu(x, y)}{\partial y^{\gamma}}+\frac{\partial^{2} \mu(x, y)}{\partial x^{2}}+5 \mu(x, y)=0,
$$

with proper initial value

$$
\mu(x, 0)=x
$$

Thus the result of Eq. (32) is defined by

$$
\mu(x, y)=x\left(1-\frac{5 y^{\gamma}}{\Gamma(\gamma+1)}+\frac{25 y^{2 \gamma}}{\Gamma(2 \gamma+1)}-\frac{125 y^{3 \gamma}}{\Gamma(3 \gamma+1)}+\frac{625 y^{4 \gamma}}{\Gamma(4 \gamma+1)}+\cdots\right)
$$

The exact result is

$$
\mu(x, y)=x \cos \sqrt{5} y .
$$

Figure 4 depicts solutions in the two-dimensional plot of exact and HPETM solutions in Figs. (a) and (b) for different values of $\gamma=2,1.9,1.8,1.7,1.6,1.5$ for $x \in[0 ; 1]$ and $y=1$. In Fig. 5, the three-dimensional plots of exact and HPETM solutions in Figs. (a) and (b), respectively, at $\gamma=2$ and the closed contact of the exact and HPETM solutions are analyzed. In Fig. 6, the graphs (c) and (d) represent the HPETM solutions at $\gamma=1.8$ and 1.6, respectively, of Example 2. The fractional-order results are found to be convergent to
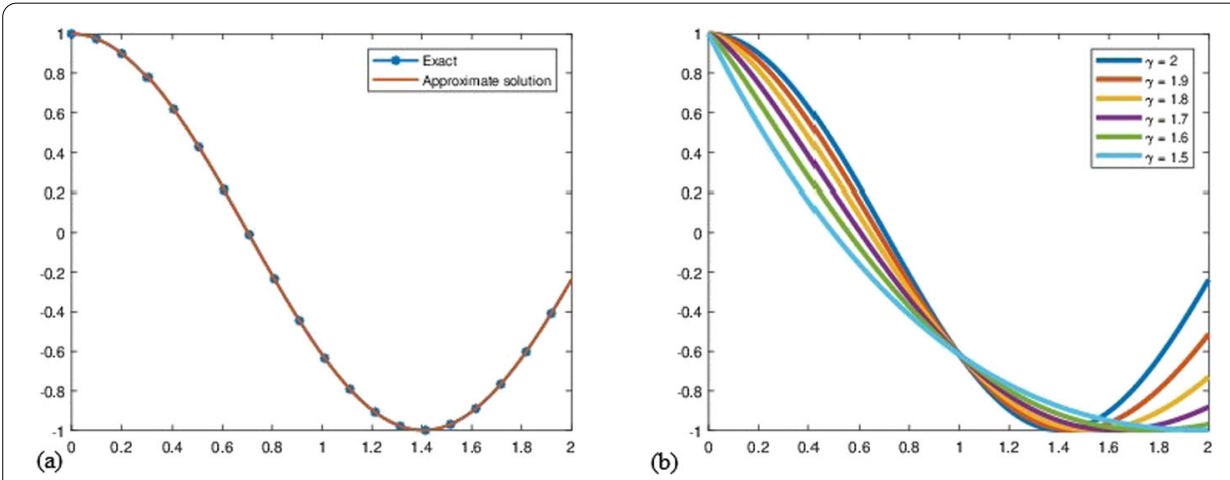

Figure 4 (a) Graph of exact and approximate solution and (b) different fractional-order results of $\gamma$ of Example 2 


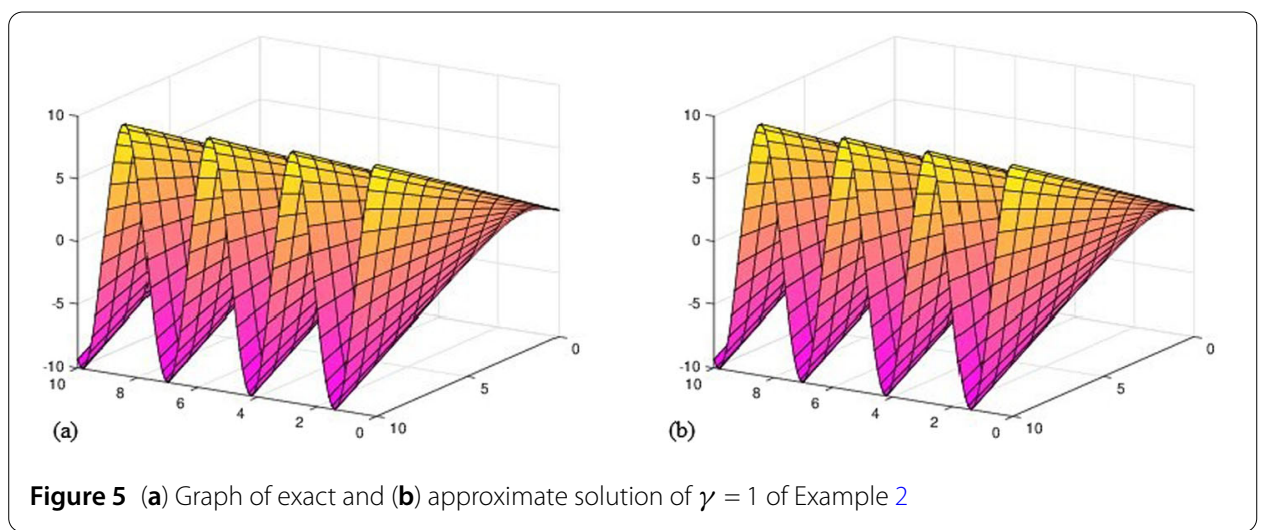

Figure 5 (a) Graph of exact and (b) approximate solution of $\gamma=1$ of Example 2

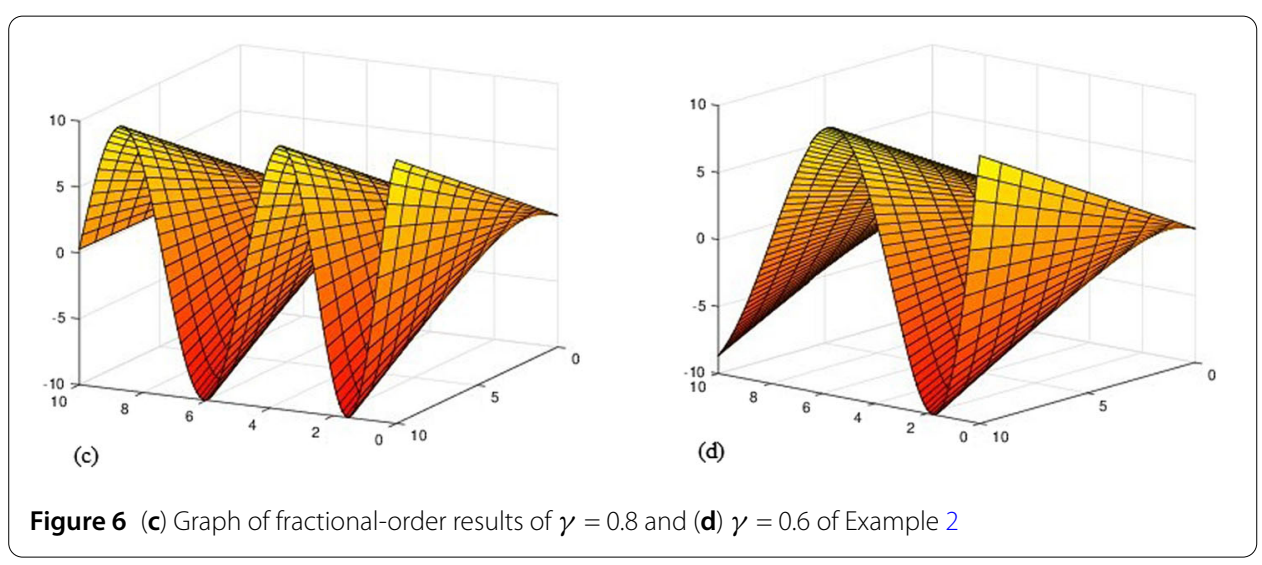

an integer-order result of the problem. In the same way, we can draw the graphs for the $y$-space fractional-order derivative.

Example 3 Consider the fractional-order Helmholtz equation

$$
\frac{\partial^{\gamma} \mu(x, y)}{\partial x^{\gamma}}+\frac{\partial^{2} \mu(x, y)}{\partial y^{2}}-2 \mu(x, y)=\left(12 x^{2}-3 x^{4}\right) \sin y, \quad 1<\gamma \leq 2,0 \leq y \leq 2 \pi,
$$

with initial conditions

$$
\mu(0, y)=0 \quad \text { and } \quad \mu_{x}(0, y)=0 .
$$

Implementing the Elzaki transformation of Eq. (35), we get

$$
\begin{aligned}
& \frac{1}{s^{\gamma}} E[\mu(x, y)]=\mu(0, y) s^{2-\gamma}-E\left\{\frac{\partial^{2} \mu(x, y)}{\partial y^{2}}-2 \mu(x, y)\right\}, \\
& E[\mu(x, y)]=s^{2} \mu(0, y)-s^{\gamma} E\left\{\frac{\partial^{2} \mu(x, y)}{\partial y^{2}}-2 \mu(x, y)\right\} .
\end{aligned}
$$

Using the inverse Elzaki transformation, we have

$$
E[\mu(x, y)]=\left(x^{4}-\frac{x^{6}}{10}\right) \sin y-E^{-1}\left[s^{\gamma} E\left\{\frac{\partial^{2} \mu(x, y)}{\partial y^{2}}-2 \mu(x, y)\right\}\right]
$$


Using HPM as in Eq. (39), we get

$$
\sum_{k=0}^{\infty} p^{k} \mu_{k}(x, y)=y-p\left[E^{-1}\left\{s^{\gamma} E\left\{\left(\sum_{k=0}^{\infty} p^{k} \mu_{k}(x, y)\right)_{y y}-2 \sum_{k=0}^{\infty} p^{k} \mu_{k}(x, y)\right\}\right\}\right] .
$$

On both sides on comparing the coefficients $p$, we obtain

$$
\begin{aligned}
& p^{0}: \mu_{0}(x, y)=\left(x^{4}-\frac{x^{6}}{10}\right) \sin y, \\
& p^{1}: \mu_{1}(x, y)=-E^{-1}\left[s^{\gamma} E\left\{\frac{\partial^{2} \mu_{0}(x, y)}{\partial y^{2}}-2 \mu_{0}(x, y)\right\}\right] \\
& =3\left(\frac{x^{\gamma+4}}{\Gamma(\gamma+5)}-\frac{72 x^{\gamma+6}}{\Gamma(\gamma+7)}\right) \sin y \text {, } \\
& p^{2}: \mu_{2}(x, y)=-E^{-1}\left[s^{\gamma} E\left\{\frac{\partial^{2} \mu_{1}(x, y)}{\partial y^{2}}-2 \mu_{1}(x, y)\right\}\right] \\
& =3\left(\frac{x^{2 \gamma+4}}{\Gamma(2 \gamma+5)}-\frac{216 x^{2 \gamma+6}}{\Gamma(2 \gamma+7)}\right) \sin y \text {, } \\
& p^{3}: \mu_{3}(x, y)=-E^{-1}\left[s^{\gamma} E\left\{\frac{\partial^{2} \mu_{2}(x, y)}{\partial y^{2}}-2 \mu_{2}(x, y)\right\}\right] \\
& =3\left(\frac{x^{3 \gamma+4}}{\Gamma(3 \gamma+5)}-\frac{648 x^{3 \gamma+6}}{\Gamma(3 \gamma+7)}\right) \sin y \text {, } \\
& p^{4}: \mu_{4}(x, y)=-E^{-1}\left[s^{\gamma} E\left\{\frac{\partial^{2} \mu_{3}(x, y)}{\partial y^{2}}-2 \mu_{3}(x, y)\right\}\right] \\
& =3\left(\frac{x^{4 \gamma+4}}{\Gamma(4 \gamma+5)}-\frac{1944 x^{2 \gamma+6}}{\Gamma(2 \gamma+7)}\right) \sin y \text {, }
\end{aligned}
$$

The series form a solution of the given example,

$$
\begin{aligned}
\mu(x, y)= & \mu_{0}(x, y)+\mu_{1}(x, y)+\mu_{2}(x, y)+\mu_{3}(x, y)+\mu_{4}(x, y)+\cdots \\
\mu(x, y)= & \left(x^{4}-\frac{x^{6}}{10}\right) \sin y+3\left(\frac{x^{\gamma+4}}{\Gamma(\gamma+5)}-\frac{72 x^{\gamma+6}}{\Gamma(\gamma+7)}\right) \sin y \\
& +3\left(\frac{x^{2 \gamma+4}}{\Gamma(2 \gamma+5)}-\frac{216 x^{2 \gamma+6}}{\Gamma(2 \gamma+7)}\right) \sin y \\
& +3\left(\frac{x^{3 \gamma+4}}{\Gamma(3 \gamma+5)}-\frac{648 x^{3 \gamma+6}}{\Gamma(3 \gamma+7)}\right) \sin y \\
& +3\left(\frac{x^{4 \gamma+4}}{\Gamma(4 \gamma+5)}-\frac{1944 x^{2 \gamma+6}}{\Gamma(2 \gamma+7)}\right) \sin y+\cdots .
\end{aligned}
$$

The exact result of Example 3, when $\gamma=2$, is

$$
\mu(x, y)=x^{4} \sin y .
$$



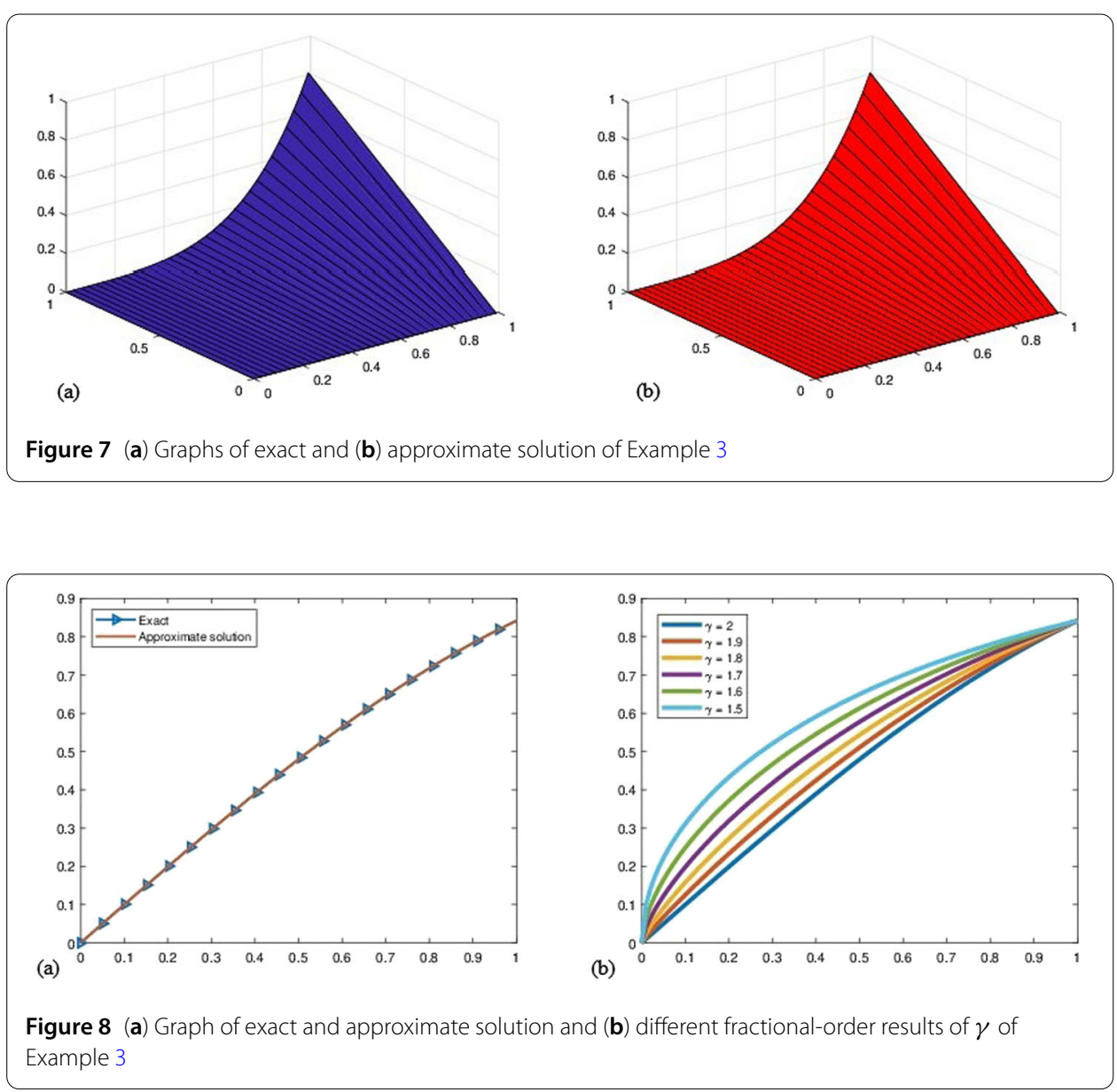

In Fig. 7, the three-dimensional plots of exact and HPETM solution in Figs. (a) and (b), respectively, at $\gamma=2$ and the closed contact of the exact and HPETM solutions are analyzed. In Fig. 8, we depict solutions in two-dimensional plots of exact and HPETM solutions in Figs. (a) and (b) for different values of $\gamma=2,1.9,1.8,1.7,1.6,1.5$ for $x \in[0 ; 1]$ and $y=1$. The fractional results are investigated and found to be convergent to an integerorder result of the problem. In the same way, we can draw the graphs for the $y$-space fractional-order derivative.

\section{Conclusions}

In this work, we utilized the homotopy perturbation Elzaki transform method to solve fractional-order Helmholtz equations and their approximate solutions were obtained. The homotopy perturbation Elzaki transform method was proved to be an effective approach for solving partial differential equations with Caputo operators due to the excellent agreement between the obtained approximate solution and the exact solution. A comparison was made to show that the method has a small computation size compared to the computational size required in other numerical methods. And its rapid convergence shows that the procedure is reliable and introduces a significant improvement in solving linear and non-linear fractional-order partial differential equations. 


\section{Acknowledgements}

This work was supported by a Korea Institute of Energy Technology Evaluation and Planning (KETEP) grant funded by the Korea government (MOTIE) (20202020900060, the Development and Application of Operational Technology in Smart Farm Utilizing Waste Heat from Particulates Reduced Smokestack).

\section{Funding}

Not applicable

\section{Availability of data and materials}

Not applicable.

\section{Competing interests}

The authors declare that they have no competing interests.

\section{Authors' contributions}

The authors declare that this study was accomplished in collaboration with the same responsibility. All authors read and approved the final manuscript.

\section{Author details}

${ }^{1}$ Informetrics Research Group, Ton Duc Thang University, Ho Chi Minh City, Vietnam. ${ }^{2}$ Faculty of Mathematics \& Statistics, Ton Duc Thang University, Ho Chi Minh City, Vietnam. ${ }^{3}$ Department of Mathematics, College of Science and Humanities in Al-Kharj, Prince Sattam bin Abdulaziz University, P.O. Box 83, Al-Kharj, 11942, Saudi Arabia. ${ }^{4}$ Department of Basic Engineering Science, Faculty of Engineering, Menoufia University, Shebin El-Kom, 32511, Egypt. ${ }^{5}$ Department of Mathematics, Faculty of Science, University of Tabuk, P.O. Box 741, Tabuk 71491, Saudi Arabia. ${ }^{6}$ Department of Mechanical Engineering, Sejong University, Seoul 05006, Korea.

\section{Publisher's Note}

Springer Nature remains neutral with regard to jurisdictional claims in published maps and institutional affiliations.

Received: 17 September 2020 Accepted: 7 December 2020 Published online: 07 January 2021

\section{References}

1. Baleanu, D., Guvenc, Z.B., Tenreiro Machado, J.A. (eds.): New Trends in Nanotechnology and Fractional Calculus Applications Springer, New York (2010)

2. Baleanu, D., et al.: Fractional Calculus: Models and Numerical Methods, vol. 3. World Scientific, Singapore (2012)

3. Baleanu, D., Tenreiro Machado, J.A., Luo, A.C.J. (eds.): Fractional Dynamics and Control Springer, Berlin (2011)

4. Jajarmi, A., Baleanu, D.: A new iterative method for the numerical solution of high-order non-linear fractional boundary value problems. Front. Phys. 8, 220 (2020)

5. Jajarmi, A., Baleanu, D.: On the fractional optimal control problems with a general derivative operator. Asian J. Control (2019). https://doi.org/10.1002/asjc.2282

6. Baleanu, D., Jajarmi, A., Mohammadi, H., Rezapour, S.: A new study on the mathematical modelling of human liver with Caputo-Fabrizio fractional derivative. Chaos Solitons Fractals 134, 109705 (2020)

7. Baleanu, D., Etemad, S., Rezapour, S.: A hybrid Caputo fractional modeling for thermostat with hybrid boundary value conditions. Bound. Value Probl. 2020(1), 64 (2020)

8. Baleanu, D., Mousalou, A., Rezapour, S.: On the existence of solutions for some infinite coefficient-symmetric Caputo-Fabrizio fractional integro-differential equations. Bound. Value Probl. 2017(1), 145 (2017)

9. Amara, A., Etemad, S., Rezapour, S.: Topological degree theory and Caputo-Hadamard fractional boundary value problems. Adv. Differ. Equ. 2020(1), 369 (2020)

10. Gupta, P.K., Yildirim, A., Rai, K.N.: Application of He's homotopy perturbation method for multi-dimensional fractional Helmholtz equation. Int. J. Numer. Methods Heat Fluid Flow 22(4), 424-435 (2012)

11. Zheng, Q., Xie, F., Lin, W.: Solution of two-dimensional Helmholtz equation by multipole theory method. J. Electromagn. Waves Appl. 13, 205-220 (1999)

12. Thompson, L.L., Pinsky, P.M.: A Galerkin least-squares finite element method for the two-dimensional Helmholtz equation. Int. J. Numer. Methods Eng. 38, 371-397 (1995)

13. Zhang, W., Dai, Y.: Finite-difference solution of the Helmholtz equation based on two domain decomposition algorithms. J. Appl. Math. Phys. 1, 18-24 (2013)

14. Samuel, M., Thomas, A.: On fractional Helmholtz equations. Fract. Calc. Appl. Anal. 13, 295-308 (2010)

15. Baleanu, D., Jassim, H.K., Al Qurashi, M.: Solving Helmholtz equation with local fractional derivative operators. Fractal Fract. 3, 43 (2019)

16. Abuasad, S., Moaddy, K., Hashim, I.: Analytical treatment of two-dimensional fractional Helmholtz equations. J. King Saud Univ., Sci. 31, 659-666 (2019)

17. Prakash, A., Goyal, M., Gupta, S.: Numerical simulation of space-fractional Helmholtz equation arising in seismic wave propagation, imaging and inversion. Pramana 93, 28 (2019)

18. Momani, S., Abuasad, S.: Application of He's variational iteration method to Helmholtz equation. Chaos Solitons Fractals 27, 1119-1123 (2006)

19. He, J.H.: Homotopy perturbation method: a new nonlinear analytical technique. Appl. Math. Comput. 135, 73-79 (2003)

20. He, J.H.: Homotopy perturbation method for bifurcation of nonlinear problems. Int. J. Nonlinear Sci. Numer. Simul. 6 207-208 (2005)

21. He, J.H.: Homotopy perturbation method for solving boundary value problems. Phys. Lett. A 350, $87-88$ (2006) 
22. He, J.H.: The homotopy perturbation method for nonlinear oscillators with discontinuities. Appl. Math. Comput. 151, 287-292 (2004)

23. He, J.H.: Application of homotopy perturbation method to nonlinear wave equations. Chaos Solitons Fractals 26, 695-700 (2005)

24. Elzaki, T.M.: The new integral transform 'Elzaki transform'. Glob. J. Pure Appl. Math. 7(1), 57-64 (2011)

25. Alshikh, A.: A comparative study between Laplace transform and two new integrals "Elzaki" transform and "Aboodh" transform. Pure Appl. Math. J. 5, 145-150 (2016)

26. Elzaki, T., Alkhateeb, S.: Modification of Sumudu transform "Elzaki transform" and Adomian decomposition method. Appl. Math. Sci. 9, 603-611 (2015)

27. Jena, R., Chakraverty, S.: Solving time-fractional Navier-Stokes equations using homotopy perturbation Elzaki transform. SN Appl. Sci. 1, 16 (2018)

28. Mahgoub, M., Sedeeg, A.: A comparative study for solving nonlinear fractional heat-like equations via Elzaki transform. Br. J. Math. Comput. Sci. 19, 1-12 (2016)

29. Das, S., Gupta, P.: An approximate analytical solution of the fractional diffusion equation with absorbent term and external force by homotopy perturbation method. Z. Naturforsch. A 65, 182-190 (2010)

30. Singh, P., Sharma, D.: Comparative study of homotopy perturbation transformation with homotopy perturbation Elzaki transform method for solving nonlinear fractional PDE. Nonlinear Dyn. 9, 60-71 (2019)

\section{Submit your manuscript to a SpringerOpen ${ }^{\circ}$} journal and benefit from:

- Convenient online submission

- Rigorous peer review

- Open access: articles freely available online

- High visibility within the field

- Retaining the copyright to your article

Submit your next manuscript at $\gg$ springeropen.com 